\title{
The Relationship between Type D Personality and Suicidality in Low-Income, Middle-Aged Adults
}

\author{
Dae Hyun Yoon', Seog Ju Kim², Jong-Ha Lee ${ }^{3}$, Pyo-Min Kim4, Doo-Heum Park ${ }^{5}$, \\ Seung Ho Ryu ${ }^{5}$, Jaehak $\mathrm{Yu}^{5}$, and Jee Hyun $\mathrm{Ha}^{5} \bowtie$ \\ 1'Department of Psychiatry, Gangnam Center, Seoul National University Hospital, Seoul, Republic of Korea \\ ${ }^{2}$ Department of Medicine, School of Medicine, Seoul National University, Seoul, Republic of Korea \\ ${ }^{3}$ Department of Social Welfare, Induk University, Seoul, Republic of Korea \\ ${ }^{4}$ Department of Social Welfare, Soongsil University, Seoul, Republic of Korea \\ ${ }^{5}$ Department of Psychiatry, School of Medicine, Konkuk University, Seoul, Republic of Korea
}

Objective Low-income adults are considered to be a group at high risk for suicide. We sought to examine the effect of type D personality and other socio-demographic factors on suicidality in low-income, middle-aged Koreans.

Methods In total, 306 low-income, middle-aged Koreans [age: 49.16 \pm 5.24 (40-59) years, 156 males, 150 females] were enrolled from the Korean National Basic Livelihood Security System. Socio-demographic data, including employment status, income, health, marital status, and educational attainment, were gathered. Beck's 19-item Scale for Suicidal Ideation (SSI) was applied to evaluate suicidality, and the DS14 was used to assess type D personality.

Results Unemployment $(\mathrm{p}<0.01)$ and absence of spouse $(\mathrm{p}=0.03)$ predicted higher SSI scores independent of other socioeconomic factors. All type D personality scores [i.e., negative affectivity (NA), social inhibition (SI), and total score] predicted higher SSI scores independent of all socioeconomic factors (all, $\mathrm{p}<0.001$ ). Subjects with type D personality had higher SSI scores $(\mathrm{p}<0.001)$, and the association between suicidality and socio-demographic factors (employment or physical health) could be found only in subjects without type D personality.

Conclusion Type D personality was a risk factor for suicide in low-income Koreans, independently from socio-economic factors. In addition, the socio-demographic factors were less prominently associated with suicidality in those with type D personality.

Psychiatry Investig 2015;12(1):16-22

Key Words Suicide, Low-income, Type D personality.

\section{INTRODUCTION}

Socioeconomic status (SES) is considered an important risk factor for suicide, and it has been shown that lower SES groups have higher suicide rates. ${ }^{1}$ Economic stressors are the second most important risk factor for committing suicide, ${ }^{2}$ and unemployment has been reported to increase suicide rates by 4 to 5-fold. ${ }^{3,4}$ In addition, lower education, physical illness, and economic inequality were also shown to be associated with

Received: October 15, 2013 Revised: February 17, 2014

Accepted: March 11, 2014 Available online: January 12, 2015

$\triangle$ Correspondence: Jee Hyun $\mathrm{Ha}, \mathrm{MD}, \mathrm{PhD}$

Department of Psychiatry, School of Medicine, Konkuk University, 120-1 Neungdong-ro, Gwangjin-gu, Seoul 143-729, Republic of Korea

Tel: +82-2-2030-7569, Fax: +82-2-2030-7748, E-mail: jhnha@naver.com

(a) This is an Open Access article distributed under the terms of the Creative Commons Attribution Non-Commercial License (http://creativecommons.org/licenses/bync/3.0) which permits unrestricted non-commercial use, distribution, and reproduction in any medium, provided the original work is properly cited. higher suicide rates. ${ }^{5}$

For many years, Korea has had the highest suicide rate of the OECD countries, and the suicide rate in Korea has increased 2 -fold in the last $10{ }^{6}$ Suicide began to increase in the late 1990s when the Korean economy suffered a financial crisis, and the most common cause of suicide reported by bereaved family members in Korea was financial hardship. ${ }^{6}$ Thus, increasing economic inequality and polarization may explain the increasing suicide rate over the last few decades. ${ }^{5,7}$

From this viewpoint, low-income Koreans constitute a specific group with a high risk for suicide. To establish an efficient suicide prevention strategy, factors associated with suicidality should be identified in high-risk populations. Furthermore, correlates of suicidality among those with low incomes may differ from those in other socioeconomic groups, and it is possible that the effects of income or employment on suicidality could be either weaker (because of a ceiling effect) or stronger 
(because of higher importance) within this low-income population.

In addition, the relationship between low income and high suicidality may be more pronounced in the middle-aged population, which is usually economically active. In Korea, suicide was the second leading cause of death in adults in their 40 s and 50s. Low income was reported to increase suicide-related behavior in China. ${ }^{8}$ In addition, low income was reported to be a major determinant of suicide mortality in middle aged Japanese. ${ }^{9}$ However, there have been very few studies on suicidality among low-income, middle-aged adults.

Although socio-demographic factors contribute to higher rates of suicide, individual risk factors may also be important. For example, personality traits may be either predisposing or protective factors for suicidality. ${ }^{10-12}$

Most mental illnesses known to increase suicide risk were state-dependent and would be change with individual condition and environment. It is possible that underlying long-term emotional condition such as traits or temperament might affect suicidality besides state-dependent factors. ${ }^{10}$ Personality trait would be an independent predictor of suicide attempt controlling other known risk factors. ${ }^{13}$ Combination of state and trait would light the more accurate prediction of suicide risk. Besides current mental illnesses needing urgent intervention, the long standing personality or trait might have a value in evaluation of suicide risk. Assessing type D personality as a trait marker could be one of the valuable tools for screening suicide risk, as it is short and easy to apply.

Type D personality was proposed in the search for the personality trait vulnerable to cardiovascular diseases. ${ }^{14}$ As the relationship between cardiovascular diseases and type A personality (competitive and time-urgent trait) has not been conclusive, Type D personality was alternatively introduced decades later. ${ }^{14}$ Type $\mathrm{D}$ personality is characterized by a tendency to experience increased negative emotion (negative affectivity, NA) and to inhibit the expression of these emotions in social situations (social inhibition, SI). Type D personality has been reported to be associated not only with cardiovascular diseases, ${ }^{14,15}$ but also with psychiatric problems such as anxiety, somatization, posttraumatic stress disorder, and depression. ${ }^{16,17}$ Although type D personality has similarity with the existing personality dimension as five-factor model, ${ }^{17}$ the synergy between negative affectivity and social inhibition confirms the discriminant validity of type D personality. ${ }^{18}$

Although major depressive disorder has been regarded as the most important risk factor of suicide, its symptoms are also state-dependent. ${ }^{13}$ For long-term traits, NA were associated with suicidality. ${ }^{19,20}$ In addition, interpersonal anxiety and social inadequacy feeling were also associated with suicide risk. ${ }^{21} \mathrm{NA}$ and SI are two major components of type D personality. In addition, many psychiatric disorders with high suicidal risk have been reported to be associated with type D personality. ${ }^{16,17}$ Therefore, although type D personality has been used in the subjects with cardiovascular disorders, it could be also used to identify the relatively long term stable trait of suicidality regardless of cardiovascular disorders.

Recently, one study reported that type D personality correlates with suicidal ideation in the general population. ${ }^{22}$ Moreover, the relationship between type $\mathrm{D}$ personality and suicidality may differ among socioeconomic classes, although most suicide studies in low-income populations were based on aggregate data, for which personality traits could not be easily explored. It also remains unclear whether type $\mathrm{D}$ personality interacts with the relationship between socio-demographic factors and suicidality.

The specific aims of the current study were to examine the association between type D personality and suicidality in lowincome, middle-aged Koreans. We also sought to explore the influence of type D personality on the relationship between socio-demographic characteristics and suicidality in this population. We hypothesized that type $\mathrm{D}$ personality would be associated with suicidality and that it would interact with the relationship between socio-demographic characteristics and suicidality among low-income, middle-aged Koreans.

\section{METHODS}

\section{Subjects}

Subjects were enrolled from Seongbuk District (Seongbukgu), Seoul, Republic of Korea. The total registered population of Seongbuk District was 489,227 inhabitants. Of these subjects, 10,153 were assisted by Korea's National Basic Livelihood Security System. Individuals who earn less than 130\% of the minimum cost of living and have no valuable assets are eligible for these benefits, and it is estimated that $3 \%$ of the general population meets this requirement. In total, 7,112 subjects were over age 65 , and 3,041 subjects were 20 to 64 years old. We selected 306 middle-aged adults (range=40-59; mean= 49.16 \pm 5.24 ; male $=156$ and female $=150$ ) using multi-stage stratified random sampling. The parent population was stratified into subgroups based on three categories; age, sex and neighborhood (dong). After determining the subgroups, subjects for each subgroup were randomly selected in line with the demographic proportion of the parent population. Among the 3,041 subjects, our target of enrollment was 300 (10\% of total subjects). We originally randomly selected 300 subjects as the first draft group and contacted them by phone call. Among them, 30 subjects (10\%) refused to participate and excluded from the study. After that exclusion, we selected extra 36 subjects from initial population using a same method. With 
multi-stage stratified random sampling, our selected sample could retain the representativeness of the parent population.

Trained interviewers visited the participants at home and performed semi-structured interviews after obtaining informed consent. It took an average of 30 minutes to complete the interview. Subjects were informed that their anonymity would be preserved that they are willing not to participate in the survey. The study was reviewed and approved by the Institutional Review Board of Konkuk Univeristy Hospital.

\section{Questionnaires}

Demographic data, including sex, age, marital status, years of education, average monthly income, and job status, were gathered. Subjects were asked if they had hypertension, arthritis, diabetes mellitus, liver disease, kidney disease, vascular disease, respiratory disorders, gastrointestinal disorders, ophthalmologic disorders, auditory disorders, stroke, or dental problems. This information was verified, and the total number of medical disorders was summed.

To measure the severity of suicidal intent, the study participants were asked to complete the Korean version of Beck's 19item Scale for Suicidal Ideation (SSI). ${ }^{23,24}$ Beck developed this questionnaire that consists of 19 items with a 3-point Likert scale to evaluate the intensity of suicide ideation. ${ }^{23}$ The questionnaire asks about symptoms that were present over the previous 7 days. Each item is scored between 0 and 2, and the total score ranges from 0 to 38 ; higher scores indicate greater suicidal intent. There is no formal cut-off score to indicate serious risk of suicidal behavior. The Korean version of the SSI was validated in a previous study, in which the internal consistency (Cronbach's alpha) value was $0.89 .^{24}$

Type D personality represents a specific personality structure characterized by the negative affect and the propensity to inhibit self-expression in social interaction, and it has been associated with an increased risk of various medical conditions and psychopathology. In our study, type D personality was measured using the DS14, ${ }^{25}$ a 14 -item self-rating questionnaire that uses a 5-point Likert scale ranging from 0 to 4 , with total scores ranging from 0 to 56 . The DS14 consists of two subscales: NA and SI. Participants who scored high on both NA and SI, using a cut-off value $\geq 10$ on each scale, were classified as having type D personality. The Korean standardized version was used, and its internal consistency was 0.88 for NA and 0.89 for SI (Cronbach's alpha). ${ }^{26}$

\section{Statistical analysis}

Group differences in continuous variables were examined using independent t-tests or ANCOVA (covariates: age and sex). Between-group comparisons of categorical data were assessed using the chi-square test. Relationships between other continuous variables were assessed using Pearson's correlation coefficients. To detect independent predictors of suicidality, multiple regression models were used (models 1 and 2). In model 1, the dependent variable was the SSI score, and the independent variables consisted of the socio-demographic variables, age, and sex. In model 2, the DS14 scores for assessing type D personality were included as 3 additional independent variables. All statistical analyses were performed with PASW statistics 17 (former SPSS, Chicago, IL, USA), and a $p$ value $<0.05$ was considered to indicate a significant difference. All tests were two-tailed.

\section{RESULTS}

\section{Characteristics of the study subjects}

The summary statistics concerning the demographic characteristics and questionnaire data for all study participants $(n=306)$ are presented in Table 1 . The study participants ranged in age from 40-59 years, with a mean age of $49.16 \pm$ 5.24 years. There were 156 male participants (50.5\%) and 150 female participants (49.5\%). The average monthly income of all participants was $632,800 \pm 352,240$ Korean won (equivalent

Table 1. Characteristics of the study subjects $(\mathrm{N}=306)$

\begin{tabular}{lc}
\hline & Mean \pm SD $(\mathrm{N}, \%)$ \\
\hline Age & $49.16 \pm 5.24$ \\
Sex & \\
Male & $156(51.0)$ \\
Female & $150(49.0)$ \\
Marital status & \\
Unmarried & $56(18.3)$ \\
Married & $80(26.1)$ \\
Divorced/Separated & $142(46.4)$ \\
Bereaved & $25(8.2)$ \\
No answer/Other & $3(1.0)$ \\
Educational attainment (years) & $10.02 \pm 3.83$ \\
Employment status & \\
Employed & $74(24.2)$ \\
Unemployed & $229(74.8)$ \\
No answer/Other & $3(1.0)$ \\
Monthly income (Korean won) & $634,200 \pm 353,350$ \\
Number of physical illnesses & $2.17 \pm 2.07$ \\
Scale for Suicidal Ideation (SSI) score & $7.57 \pm 7.12$ \\
Type D personality & \\
Score for Negative Affectivity (NA) & $11.53 \pm 8.48$ \\
Score for Social Inhibition (SI) & $10.37 \pm 8.95$ \\
Score for type D personality & $21.90 \pm 16.94$ \\
Proportion of subjects with type D & $130(42.5)$ \\
personality & \\
\hline
\end{tabular}


to USD 589.20). There were 82 married (26.2\%), 56 unmarried (17.9\%), 147 divorced or separated (47.0\%), and 25 widowed $(8.0 \%)$ participants. The mean educational attainment was $10.03 \pm 3.82$ years. The majority of subjects were unemployed ( $n=234,74.8 \%)$. Most participants $(n=257,84.0 \%)$ suffered from at least one physical illness, and 158 (51.6\%) had more than one physical illness. The mean number of physical illnesses reported was $2.2 \pm 2.0$.

There were no gender differences in age, educational attainment, monthly income, number of physical illness, or marital status. However, unemployment was more common in men $(81.17 \%)$ than in women $(69.80 \%)$. Older age was associated with higher rates of unemployment $(\mathrm{t}=3.50, \mathrm{p}<0.01)$, less education $(\mathrm{r}=-0.169, \mathrm{p}<0.01)$, lower monthly income $(\mathrm{r}=-0.238$, $\mathrm{p}<0.001)$, and more physical illnesses $(\mathrm{r}=0.263, \mathrm{p}<0.001)$. There was no significant difference in age between marital statuses, although married subjects had higher income ( $t=5.39$, $\mathrm{p}<0.001)$ and fewer physical illnesses $(\mathrm{t}=2.29, \mathrm{p}<0.05)$. Unemployed subjects had lower income $(\mathrm{t}=5.25, \mathrm{p}<0.001)$ and more physical illness ( $\mathrm{t}=7.04, \mathrm{p}<0.001)$, whereas higher monthly income correlated with greater educational attainment $(\mathrm{r}=0.214$, $\mathrm{p}<0.001)$ and fewer physical illnesses $(\mathrm{r}=-0.276, \mathrm{p}<0.001)$.

\section{Socio-demographic variables and suicidality}

The relationship between socio-demographic characteristics and SSI score is summarized in Table 2. The SSI score was negatively correlated with educational attainment $(\mathrm{r}=-0.138$, $\mathrm{p}<0.05)$ and monthly income $(\mathrm{r}=-0.135, \mathrm{p}<0.05)$ but was positively correlated with the number of physical illnesses $(r=0.217$, $\mathrm{p}<0.001)$. Married and employed subjects had lower SSI scores $(\mathrm{t}=1.19, \mathrm{p}<0.05 ; \mathrm{t}=3.60, \mathrm{p}<0.001$, respectively). SSI scores were not significantly correlated with age or sex in our participants.

There was a complex and close relationship among employment, education, income, marriage, and physical health. Stepwise multiple regressions were performed to find the independent socio-demographic predictor for higher SSI score in low-income, middle-aged adults, for which the dependent variable was the SSI score and the independent variables were employment, marital status, monthly income, educational attainment, number of physical illnesses, age, and sex. In this regression model, higher SSI score was predicted by unemployment $(\beta=0.17, \mathrm{p}<0.01)$ and marital status $(\beta=0.13, \mathrm{p}<0.05)$ but not by education, income, or physical health.

\section{Type $D$ personality and suicidality}

SSI scores were positively correlated with higher NA scores $(\mathrm{r}=0.455, \mathrm{p}<0.001)$, SI scores $(\mathrm{r}=0.430, \mathrm{p}<0.001)$, and total type $\mathrm{D}$ scores $(\mathrm{r}=0.455, \mathrm{p}<0.001)$ (Table 2$)$. Type $\mathrm{D}$ scores were also correlated with lower educational attainment $(r=-0.200$, $\mathrm{p}<0.001)$, lower monthly income $(\mathrm{r}=-0.300, \mathrm{p}<0.001)$, and physical health $(\mathrm{r}=0.340, \mathrm{p}<0.001)$. Type $\mathrm{D}$ scores were higher in subjects without spouse $(\mathrm{t}=4.02, \mathrm{p}<0.001)$ and unemployed subjects $(\mathrm{t}=4.00, \mathrm{p}<0.001)$.

Because there was a significant relationship between type

Table 2. The relationship between suicidality, socioeconomic variables, and type $\mathrm{D}$ personality

\begin{tabular}{|c|c|c|c|}
\hline Beck's Scale for Suicidal Ideation & Mean \pm SD & tor $\mathrm{r}$ & $\mathrm{p}$ \\
\hline \multicolumn{4}{|l|}{ Comparison of suicidality scores } \\
\hline \multicolumn{4}{|l|}{ Sex } \\
\hline Male & $7.60 \pm 7.00$ & $\mathrm{t}=0.06$ & 0.95 \\
\hline Female & $7.55 \pm 7.31$ & & \\
\hline \multicolumn{4}{|l|}{ Marital status } \\
\hline Married & $6.28 \pm 6.26$ & $\mathrm{t}=1.19$ & 0.05 \\
\hline Single & $7.92 \pm 6.28$ & & \\
\hline \multicolumn{4}{|l|}{ Employment status } \\
\hline Employed & $5.39 \pm 5.38$ & $\mathrm{t}=3.60$ & $<0.001$ \\
\hline Unemployed & $8.26 \pm 7.49$ & & \\
\hline \multicolumn{4}{|c|}{ Correlation between socioeconomic variables and suicidality } \\
\hline Age & & $\mathrm{r}=0.052$ & 0.36 \\
\hline Education attainment (years) & & $\mathrm{r}=0.138$ & 0.02 \\
\hline Monthly income (Korean won) & & $\mathrm{r}=-0.135$ & 0.02 \\
\hline Number of physical illnesses & & $\mathrm{r}=0.217$ & $<0.001$ \\
\hline \multicolumn{4}{|l|}{ Correlation between type $\mathrm{D}$ personality and suicidality } \\
\hline NA score & & $\mathrm{r}=0.455$ & $<0.001$ \\
\hline SI score & & $\mathrm{r}=0.430$ & $<0.001$ \\
\hline Total DS14 score & & $\mathrm{r}=0.455$ & $<0.001$ \\
\hline
\end{tabular}

NA: negative affectivity, SI: social inhibition 
D personality and socio-demographic variables, stepwise multiple regressions were performed to identify independent predictors for higher SSI scores, for which the dependent variable was SSI score and the independent variables were type D personality score (total, NA, or SI scores), employment, marital status, monthly income, educational attainment, number of physical illnesses, age, and sex. In this regression model, higher SSI scores were predicted by the type D score (total, $\beta=0.46, p<0.001 ; \mathrm{NA}, \beta=0.46, \mathrm{p}<0.001 ;$ SI, $\beta=0.44, \mathrm{p}<0.001$ ) but not by socio-demographic factors.

\section{Comparison between subjects with and without type $D$ personality}

Subjects with type $\mathrm{D}$ personality had lower educational attainment $(\mathrm{t}=3.16, \mathrm{p}<0.01)$, lower monthly income $(\mathrm{t}=4.53$, $\mathrm{p}<0.001)$, and more physical illnesses $(\mathrm{t}=4.24, \mathrm{p}<0.001)$ than did subjects without type D personality. Furthermore, employment (chi=6.94, $\mathrm{p}<0.05)$ and married status (chi=8.11, $\mathrm{p}<$ 0.01 ) were less common in subjects with type $D$ personality.

Subjects with type D personality also had higher SSI scores $(t=6.44, p<0.001)$ (Figure 1). Even after controlling for sex, age, and other socio-demographic variables, SSI scores were higher in subjects with type $\mathrm{D}$ personality $(\mathrm{F}=35.43, \mathrm{p}<0.001)$.

\section{The influence of type $\mathrm{D}$ personality on the relationship between suicidality and socio-demographic variables}

The relationship between SSI scores and socio-demographic variables was investigated separately for subjects with and without type D personality. The SSI scores of subjects without type $\mathrm{D}$ personality were significantly correlated with the number of physical illnesses $(r=0.187, \mathrm{p}<0.05)$. Among subjects without type D personality, unemployed subjects had higher

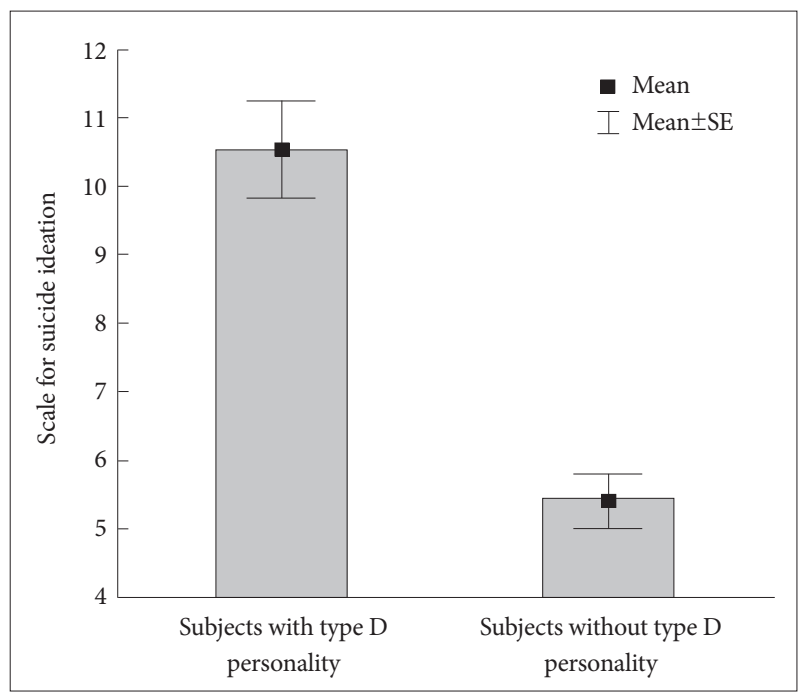

Figure 1. Suicidality of subjects with and without type $D$ personality.
SSI scores than did employed subjects $(t=2.16, p<0.05)$. SSI scores were not correlated with age, educational attainment, income, or the number of physical illnesses in subjects without type D personality. However, SSI scores were not significantly related to any socio-demographic variables in subjects with type $\mathrm{D}$ personality.

\section{DISCUSSION}

In the current study, we found that type $\mathrm{D}$ personality was related to suicidality in a economically high-risk group and that type $\mathrm{D}$ personality influenced the relationship between socio-demographic factors and suicidality. To the best of our knowledge, this study was the first to investigate the effect of type $\mathrm{D}$ personality on the association between socioeconomic factors and suicidality.

As hypothesized, type $\mathrm{D}$ personality correlated with suicidality in our low-income, high-risk group. In addition, our findings are in agreement with a previous study that reported greater suicidal ideation in subjects with type $\mathrm{D}$ personality.22 This previous study categorized the presence or absence of suicidal ideation using only one question, whereas our study assessed the degree of suicidality. In addition, our findings expand on the previous findings, as we found that personality traits are important risk factors for suicide, even in low-income, high-risk groups.

Personality characteristics that serve as risk factors for suicide include neuroticism, introversion, anxiety, aggression, impulsivity, suspiciousness, hopelessness, self-criticism, perfectionism, guilt, resentment, and irritability. Conversely, resilience, hardiness, agreeableness, and the stability of self-esteem are known protective factors against suicide. ${ }^{10}$ The SI criterion for type $\mathrm{D}$ personality refers to the persistent tendency to inhibit the expression of emotions and behaviors in social interactions; SI may also represent a consistent pattern of social interaction, similar to introversion. In addition, emotional inhibition may be related to hopelessness and isolation. The NA criterion of type D personality may include depression, anger, or anxiety, which are closely associated with suicide, ${ }^{27}$ However, our results shed no light on the nature of the causal pathway linking type $\mathrm{D}$ personality with suicidality.

A number of previous studies have reported that marital status, employment, physical health, income, and educational attainment are related to suicidal risk, ${ }^{3,5,28-32}$ and these sociodemographic factors were also associated with suicidality in the current study. However, there were complex, bidirectional relationships between these risk factors. For example, physical illness may result in unemployment and low income, while low income may result in the decreased use of necessary medical services. Thus, it is difficult to differentiate which factors 
play a key role in provoking suicidal ideation, especially in low-income populations. In the current study, unemployment and unmarried status were independent predictors for suicidal ideation, and both unemployment and unmarried status can lead to social withdrawal and loneliness. Although it remains unclear why unemployment and unmarried status were shown to have a greater independent effect on suicide as compared to other factors, loneliness may be an important mediating factor in low-income, middle-aged adults.

The SI criterion for type D personality refers to the persistent tendency to inhibit the expression of emotions and behaviors in social interactions. SI may also represent a consistent pattern of social interaction, similar to introversion. In addition, emotional inhibition may be related to hopelessness and isolation. Social inhibition trait reflects higher interpersonal anxiety and insufficiency of social relationship which were associated with suicide risk. ${ }^{21}$ The NA criterion of type D personality may include depression, anger, or anxiety, which are closely associated with suicide. ${ }^{27} \mathrm{~A}$ recent study reported that negative affectivity was a significant predictor of suicide attempts after controlling other known risk factors through 7 years of follow-up. ${ }^{13}$ In borderline personality disorder which are associated with higher risk of suicide attempt or completion, ${ }^{32}$ long term negative affectivity and impulsivity might be indicated as underlying traits. Negative affectivity has been reported to be more significant for suicide than impulsivity. ${ }^{13}$

According to our results, the presence of type $\mathrm{D}$ personality was an independent predictor for suicidality even after adjusting for confounding socio-demographic variables, most of which are strongly tied to suicidality. Furthermore, our study suggests that intrinsic factors, such as personality, and extrinsic factors, such as lower socioeconomic status, are both important to consider when assessing suicidal risk, as too great an emphasis on an external or an internal locus may interfere with developing efficient suicide prevention programs. Thus, the government should take steps to provide a welfare system and promote jobs for low-income adults in an effort to prevent suicide. In addition to these long-term social policies, screening for suicidality, especially for those with type D personality, should be performed to help low-income, high-risk groups. Moreover, individuals with type D personality may have difficulty developing a social network or asking for help.

In the current study, the association between suicidality and employment was dependent on the presence of type D personality; only subjects without type $\mathrm{D}$ personality showed an association between higher suicidality and unemployment. Although speculative, some plausible explanations may be inferred, and our findings raise the possibility that type D personality strongly induces suicidal ideation regardless of sociodemographic status. Otherwise, low-income adults with type
D personality may have strong suicidal ideation even without other negative environmental factors, as they may interpret their low-income status as an extremely negative factor. On the contrary, for those without type D personality, low income alone may not be sufficient to produce suicidal ideation. However, it remains unclear why personality affects the relationship between suicidality and employment.

Education level, income, and physical health were not related with SSI score in the study. It would be an unexpected finding considering that they have been known as risk factors for depression or suicidal idea. This discrepancy may be due to the low-income, middle aged sample of the present study. Income or education levels were mostly even or homogenous to make a significant difference between our low-income subjects. In addition, the impact of physical health on suicidality is not significant in our middle aged subjects. It may be due to the fact that physical illnesses of middle aged subjects are usually not so disable as those of elderly. On the contrary, employment and marital status predicted higher SSI because they were immediate concerns of their age. The results might imply that socio-demographic risk factors could be delineated by age-related specific concerns.

The proportion of type $\mathrm{D}$ personality was relatively higher in the current study. The proportion of type $\mathrm{D}$ personality varies from $17.3 \%$ to $67.0 \% .^{33}$ Although high prevalence of type D personality was usually found in patients with cardiovascular diseases, there was no difference between those with and without type D personality in our study.

The higher proportion of the type D personality in our middle-aged, low income subjects may be influenced by persistent negative cognitive schema after long standing lower socioeconomic status.

Of note, there were several limitations of the current study. One limitation was the use of suicidal ideation rather than suicide attempt or completion. However, ideation precedes an attempt in $95 \%$ of attempts, ${ }^{34}$ although it represents a distinct construct that differs from suicidal attempt. Therefore, suicidal ideation likely provides a reliable proxy measure for the real risk of suicide. Another limitation may have been the crosssectional nature of the study, and a longitudinal study may be needed to confirm a causal relationship between type D personality and suicidality. Another area of concern was the specificity of our low-income, middle-aged Korean population, as our findings may not be generalizable to other socio-cultural contexts.

In conclusion, we found that type $\mathrm{D}$ personality was independently associated with increased suicidality among lowincome, middle-aged Koreans. Although unemployment and unmarried status were also predictors for suicidality in this population, these factors were not significantly related to sui- 
cidality in those with type $\mathrm{D}$ personality.

\section{REFERENCES}

1. Lorant V, Kunst AE, Huisman M, Costa G, Mackenbach J; EU Working Group on Socio-Economic Inequalities in Health. Socio-economic inequalities in suicide: a European comparative study. Br J Psychiatry 2005; 187:49-54.

2. Rich CL, Warstadt GM, Nemiroff RA, Fowler RC, Young D. Suicide, stressors, and the life cycle. Am J Psychiatry 1991;148:524-527.

3. Kreitman N, Platt S. Suicide, unemployment, and domestic gas detoxification in Britain. J Epidemiol Community Health 1984;38:1-6.

4. Schony W, Grausgruber A. Epidemiological data on suicide in UpperAustria 1977-1984. Crisis 1987;8:49-52.

5. Kim MH, Jung-Choi K, Jun HJ, Kawachi I. Socioeconomic inequalities in suicidal ideation, parasuicides, and completed suicides in South Korea. Soc Sci Med 2010;70:1254-1261.

6. Korea National Statistical Office. Social indexes of Korea 2012. Seoul: Statistics Korea; 2012.

7. Ben Park BC, Lester D. Social integration and suicide in South Korea. Crisis 2006;27:48-50.

8. Ma X, Xiang YT, Cai ZJ, Li SR, Xiang YQ, Guo HL, et al. Lifetime prevalence of suicidal ideation, suicide plans and attempts in rural and urban regions of Beijing, China. Aust N Z J Psychiatry 2009;43:158-166.

9. Yamasaki A, Sakai R, Shirakawa T. Low income, unemployment, and suicide mortality rates for middle-age persons in Japan. Psychol Rep 2005;96:337-348.

10. Brezo J, Paris J, Turecki G. Personality traits as correlates of suicidal ideation, suicide attempts, and suicide completions: a systematic review. Acta Psychiatr Scand 2006;113:180-206.

11. Useda JD, Duberstein PR, Conner KR, Conwell Y. Personality and attempted suicide in depressed adults 50 years of age and older: a facet level analysis. Compr Psychiatry 2004;45:353-361.

12. Ekinci O, Albayrak Y, Ekinci AE. Temperament and character in euthymic major depressive disorder patients: the effect of previous suicide attempts and psychotic mood episodes. Psychiatry Investig 2012;9: 119-126.

13. Yen S, Shea MT, Sanislow CA, Skodol AE, Grilo CM, Edelen MO, et al. Personality traits as prospective predictors of suicide attempts. Acta Psychiatr Scand 2009;120:222-229.

14. Denollet J, Sys SU, Stroobant N, Rombouts H, Gillebert TC, Brutsaert DL. Personality as independent predictor of long-term mortality in patients with coronary heart disease. Lancet 1996;347:417-421.

15. O’Dell KR, Masters KS, Spielmans GI, Maisto SA. Does type-D personality predict outcomes among patients with cardiovascular disease? A meta-analytic review. J Psychosom Res 2011;71:199-206.

16. Pedersen SS, Denollet J. Validity of the Type D personality construct in Danish post-MI patients and healthy controls. J Psychosom Res 2004; 57:265-272.

17. De Fruyt F, Denollet J. Type D personality: a five-factor model perspec- tive. Psychol Health 2002;17:671-683.

18. Howard S, Hughes BM. Construct, concurrent and discriminant validity of Type D personality in the general population: associations with anxiety, depression, stress and cardiac output. Psychol Health 2012;27: 242-258.

19. Langhinrichsen-Rohling J, Arata C, Bowers D, O’Brien N, Morgan A. Suicidal behavior, negative affect, gender, and self-reported delinquency in college students. Suicide Life Threat Behav 2004;34:255-266.

20. Fushimi M, Sugawara J, Shimizu T. Suicide patterns and characteristics in Akita, Japan. Psychiatry Clin Neurosci 2005;59:296-302.

21. Hall RC, Platt DE, Hall RC. Suicide risk assessment: a review of risk factors for suicide in 100 patients who made severe suicide attempts. Evaluation of suicide risk in a time of managed care. Psychosomatics 1999;40:18-27.

22. Michal M, Wiltink J, Till Y, Wild PS, Münzel T, Blankenberg S, et al. Type-D personality and depersonalization are associated with suicidal ideation in the German general population aged 35-74: results from the Gutenberg Heart Study. J Affect Disord 2010;125:227-233.

23. Beck AT, Kovacs M, Weissman A. Assessment of suicidal intention: the Scale for Suicide Ideation. J Consult Clin Psychol 1979;47:343-352.

24. Shin MS, Park GB, Oh KJ, Kim JS. Study of suicide attitude in high school students; analysis of structural relationship between depressionhopelessness-suicide. Korean J Psychol 1990;9:1-19.

25. Denollet J. DS14: standard assessment of negative affectivity, social inhibition, and Type D personality. Psychosom Med 2005;67:89-97.

26. Lim HE, Lee MS, Ko YH, Park YM, Joe SH, Kim YK, et al. Assessment of the type $\mathrm{D}$ personality construct in the Korean population: a validation study of the Korean DS14. J Korean Med Sci 2011;26:116-123.

27. Capron DW, Fitch K, Medley A, Blagg C, Mallott M, Joiner T. Role of anxiety sensitivity subfactors in suicidal ideation and suicide attempt history. Depress Anxiety 2012;29:195-201.

28. Luoma JB, Pearson JL. Suicide and marital status in the United States, 1991-1996: is widowhood a risk factor? Am J Public Health 2002;92: 1518-1522.

29. Goodwin RD, Marusic A, Hoven CW. Suicide attempts in the United States: the role of physical illness. Soc Sci Med 2003;56:1783-1788.

30. Abel EL, Kruger ML. Educational attainment and suicide rates in the United States. Psychol Rep 2005;97:25-28.

31. Jungeilges J, Kirchgassner G. Economic welfare, civil liberty, and suicide: an empirical investigation. J Soc Econ 2002;31:215-231.

32. Yen S, Shea MT, Sanislow CA, Grilo CM, Skodol AE, Gunderson JG, et al. Borderline personality disorder criteria associated with prospectively observed suicidal behavior. Am J Psychiatry 2004;161:1296-1298.

33. Song EK, Son YJ. The analysis of type D personality research as a psychosocial risk factor in cardiovascular disease for elders with a chronic disease. Taehan Kanho Hakhoe Chi 2008;38:19-28.

34. Sokero TP, Melartin TK, Rytsälä HJ, Leskelä US, Lestelä-Mielonen PS, Isometsä ET. Suicidal ideation and attempts among psychiatric patients with major depressive disorder. J Clin Psychiatry 2003;64:1094-1100. 